\title{
Review of Guidelines for the Evaluation and Treatment of Leukocytospermia in Male Infertility
}

\author{
Robert J. Brunner ${ }^{1}$, Jonathan H. Demeter ${ }^{2}$ (i), Puneet Sindhwani ${ }^{2}$ (iD \\ ${ }^{1}$ College of Medicine and Life Sciences, University of Toledo, ${ }^{2}$ Department of Urology, College of Medicine and Life Sciences, University of \\ Toledo, Toledo, $\mathrm{OH}$, USA
}

\begin{abstract}
Leukocytospermia is an ill-defined and poorly understood condition affecting up to $30 \%$ of male factor infertility. Current guidelines on leukocytospermia vary significantly, although it has been linked to increased rates of infertility, uncertainty about its clinical significance, diagnosis, and treatment remains. The guidelines are conflicting with sparse data scattered across different specialties and continents. This study aims to compare and contrast available international guidelines and recommendations. In addition to these guidelines, we sought to consolidate the findings of trials over the last several decades. English language articles on human observational studies, retrospective, prospective, clinical trials and randomized control trials were searched for using the following terms: "leukocytospermia, pyospermia, and male infertility." Articles about treatment and management of leukocytospermia that were published between January 2010 and April 2018 were included, as well as four articles referenced in best practice and guideline statements from urological and andrological associations. Disagreements on this topic are highlighted as some guidelines describe no correlation between leukocytospermia and infertility while others show that treatment leads to improvement of sperm quality by many measures including improved pregnancy rate. Various treatments have been suggested including antibiotics, anti-inflammatory medications, and frequent ejaculation. There is a need for definitive characterization of Leukocytospermia as an infectious or inflammatory marker and a re-evaluation of the leukocyte concentration threshold. Additional studies investigating rates of conception as a measure of outcome are needed, to provide greater level of evidence and generalizability of leukocytopsermia management.
\end{abstract}

Keywords: Andrology; Infection; Infertility, male; Leukocytes; Semen

This is an Open Access article distributed under the terms of the Creative Commons Attribution Non-Commercial License (http://creativecommons.org/licenses/by-nc/4.0) which permits unrestricted non-commercial use, distribution, and reproduction in any medium, provided the original work is properly cited.

\section{INTRODUCTION}

Infertility, or the failure of a sexually active couple to conceive after one year of unprotected intercourse, affects approximately $15 \%$ of couples [1]. According to the American Urological Association (AUA), a male factor is the sole cause of infertility in $20 \%$ of cases and contributes in about 50\% [2,3]. While there are many etiologies for male factor infertility, infectious processes are estimated to contribute to about $15 \%$ of such cases [4].

Leukocytospermia (LCS) or pyospermia, often defined as the presence of more than one million leukocytes in $1 \mathrm{~mL}$ of semen, has been proposed as an indicator for genital tract infection and inflammation. This link remains controversial as multiple studies have not found

Received: Aug 25, 2018 Revised: Nov 20, 2018 Accepted: Dec 5, 2018 Published online Dec 31, 2018

Correspondence to: Jonathan H. Demeter iD https://orcid.org/0000-0002-3745-9005

Department of Urology, College of Medicine and Life Sciences, University of Toledo, 3000 Arlington Ave. Toledo, OH 43614, USA.

Tel: +1-419-383-3505, Fax: +1-419-383-3785, E-mail: jhdemeter@gmail.com 
a link between LCS and a confirmed genital tract infection [5]. However, there is a stronger case that the presence of LCS is a negative prognostic factor for fertility, regardless of if it truly represents an acute or chronic genital tract infection [6]. It is postulated that reactive oxygen species (ROS) generated by leukocytes are responsible for negatively affecting sperm function. It is present in up to $30 \%$ of infertile men [7]. LCS is a marker for underlying inflammation or possible infection, which negatively impacts spermatogenesis or maturation as seen by rates of infertility and semen parameters. Specifically, LCS has been linked to a worsening of many qualitative and quantitative sperm parameters, such as morphology, motility, and sperm count [8]. It should therefore be a consideration in the evaluation of many male factor infertility cases.

A systematic review of the effectiveness of antibiotics by Jung et al [9] demonstrated that the current literature is inconclusive and often fraught with bias. It may, however, hint at the promise of antibiotic therapy with regard to improved semen quality and resolution of LCS. As this review did not comment on the full management of LCS or of alternative treatment methods, we aim to do both. Specifically, we include an evaluation of the various management guidelines and subsequently compare the available trials of antibiotic therapy with those utilizing anti-inflammatory treatment as well.

Thus, the question remains: what is the clinical relevance of LCS and how should it be managed? Although an assessment of leukocytes is a part of the standard semen analysis as outlined by the World Health Organization's (WHO) laboratory manual [10], there is no consensus on guidelines for its diagnosis, clinical implications, or treatment recommendations. The purpose of our study is to review and evaluate current guidelines for the diagnosis, treatment, and management of LCS and identify how they differ internationally. We review the current guidelines of four urological and andrological bodies worldwide to compare recommendations as well as highlight areas requiring further investigation. We also review and compare observational studies and clinical trials in order to evaluate the evidence available and to provide urologists a comprehensive overview of this ill-defined and poorly understood condition.

\section{MATERIALS AND METHODS}

This report is a review of the guidelines using the following keywords: "leukocytospermia, pyospermia, prostatitis, and male infertility." To keep our review of guidelines current, articles providing clinical recommendations were excluded if published before 2010. Our criteria for "guidelines" required the collaborative contribution of authors from many institutions in a committee or conference setting.

Four urological and andrological bodies provide guidelines or best practice statements on the finding of LCS. These include the AUA, the Canadian Urological Association (CUA), American Society for Reproductive Medicine (ASRM), and the European Association of Urology (EAU). The European Society of Human Reproduction and Embryology, Urological Association of Asia, and the Japanese Urological Association had no guidelines available. We visited each respective organization's site to begin a search for LCS ("auanet. org," "cua.org," "fertstert.org," "uroweb.org," "eshre. eu," "uaanet.org," and "urol.or.jp", respectively). For the ASRM, we used the Fertility and Sterility site instead of the ASRM home site as this is where their journal articles can be found. Additionally, we searched PubMed, Embase, and Cochrane review ("ncbi.nlm.nih. gov," "embase.com," and "cochrane.org", respectively) for guidelines using the same keywords and criteria. The level of evidence for these recommendations was compared when available.

A single, most recent iteration of the guidelines article was selected for each respective organization. In cases where multiple updates were identified, the most recent article was selected while the older editions were excluded. The selected articles were then evaluated and compared according to their statements on clinical significance, diagnostic recommendations, and treatment recommendations. The evidence for the components of each publication associated with LCS were also evaluated and compared.

Subsequently, we conducted a review of observational studies and clinical trials related to LCS according to the PRISMA (Preferred Reporting Items for Systematic Reviews and Meta-Analyses) guidelines (Fig. 1). We searched PubMed, Embase, and Cochrane library using the following terms: "leukocytospermia, pyospermia, and male infertility." Our results were filtered for clinical trials and randomized control trials (RCT). 


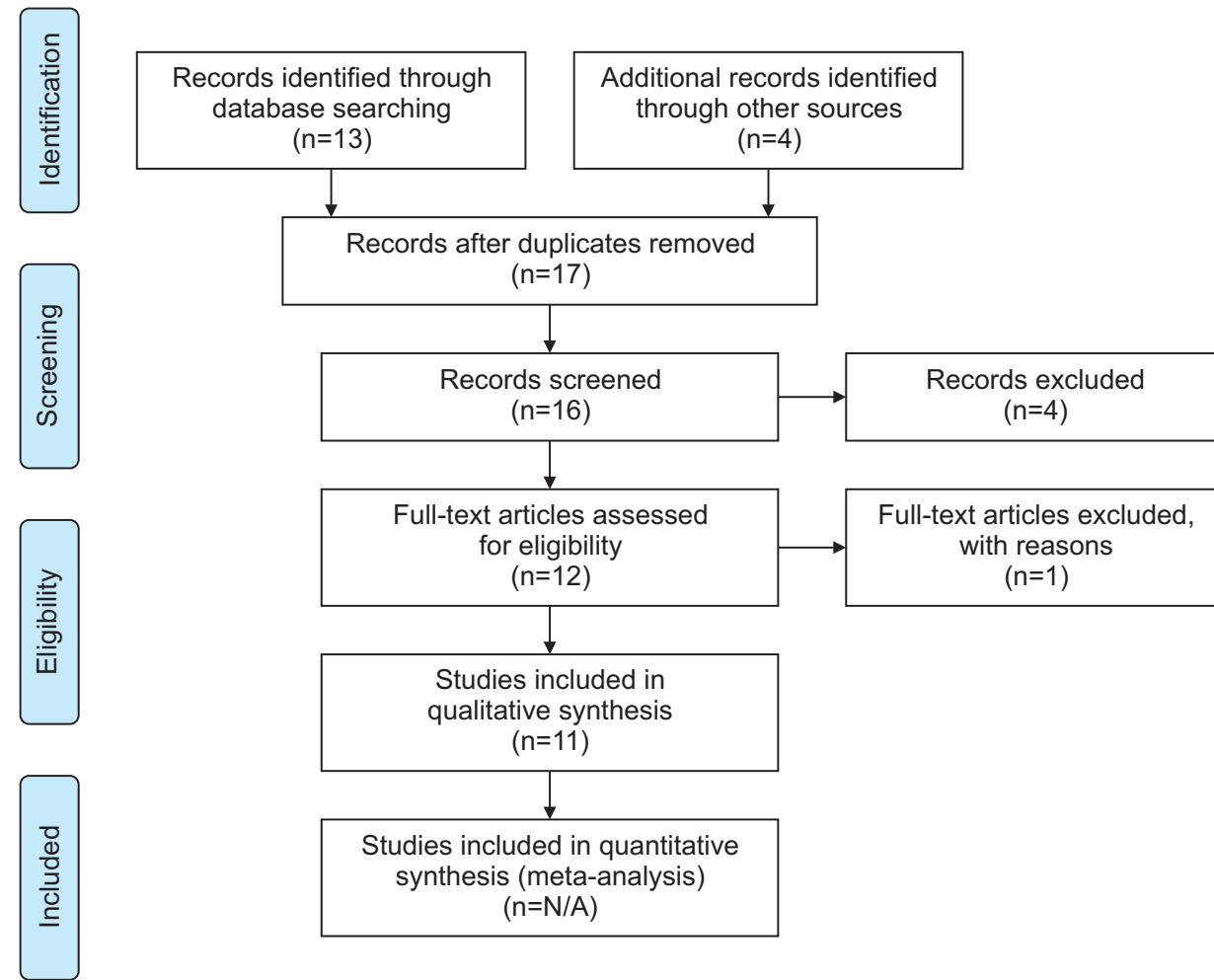

Fig. 1. Flow diagram for the systematic review of leukocytospermia clinical trials. Figure is a flow diagram depicting the search algorithm and results. PubMed, Embase, and Cochrane library was searched for English language articles on human observational studies, clinical trials and randomized control trials using the following terms: "leukocytospermia, pyospermia, and male infertility." Also included was 4 articles referenced by the various guidelines discussed in the paper. The remaining articles were screened for duplicates and non-english articles were excluded of which there was 4 . Another article was deemed ineligible as this study's seminal collection method varied so dramatically from that of the others studied. In total 11 articles are included in this review. N/A: not available.
Articles that pertained to treatment of LCS and that were published between January 1, 2010 and April 30, 2018 were included in the review. By reviewing articles from 2010 onward, we hope to capture any relevant research that may not have factored into these clinical guideline recommendations, which have been reviewed or updated in 2011 or more recently.

In addition, four articles commonly referenced in best practice and guideline statements are critically evaluated and reported on. Articles were screened for those published in English, conducted on human subjects, and for duplicates within the search. Each selected trial was evaluated based on selected patient population, diagnostic process, and the treatment effect on sperm parameters, resolution rate, and pregnancy rate.

Risk of bias was assessed at the outcome level. Special consideration was made to compare the various patient populations utilized and the presence or absence of a control group in each study in order to evaluate potential bias.

\section{RESULTS}

Table 1 summarizes the various components of the current available guidelines. It also highlights major differences and congruent interpretations at a glance. A review and comparison of the AUA, ASRM, CUA, and EAU revealed consistent recommendations for diagnostic procedures and confirmation only. However, the recommendations for the clinical interpretation and treatment of these results differ, or are entirely lacking. Each of these parameters are discussed and analyzed in detail below.

\section{American Urological Association}

In a published guideline on the optimal evaluation of male infertility [11], the AUA makes reference of LCS as a possible contributor to the infertility picture. They recommend evaluation using wet microscopy to identify round cells with confirmation of leukocytes using cytological staining or immunohistochemistry. They imply that this may indicate an underlying genital tract infection, warranting further clinical evaluation of this process. They ultimately do not see the quantification of leukocytes as a requirement in the evaluation of male infertility. Specialized tests, such as leukocyte quantification, may only be useful for a subset of patients, specifically useful in identifying factors of idiopathic male infertility or for the evaluation of assisted reproductive technology (ART) options. Unfortunately, no level of evidence classification is offered for this 
Table 1. Comparison of four major urological and andrological association's guidelines on leukocytospermia

\begin{tabular}{|c|c|c|c|}
\hline & AUA and ASRM & CUA & EAU \\
\hline Definition & $>1$ million leukocytes per $\mathrm{mL}$ semen & $>1$ million leukocytes per $\mathrm{mL}$ semen & $>1$ million leukocytes per $\mathrm{mL}$ semen \\
\hline Diagnostic method & $\begin{array}{l}\text { Wet mount microscopy confirmed } \\
\text { with immunohistochemistry }\end{array}$ & N/A & $\begin{array}{l}\text { Wet mount microscopy confirmed } \\
\text { with peroxidase positive staining }\end{array}$ \\
\hline Clinical significance & $\begin{array}{l}\text { May indicate underlying genital } \\
\text { tract infection }\end{array}$ & No clinical significance & $\begin{array}{l}\text { Indicate sign of genital tract } \\
\text { inflammation only; no direct link to } \\
\text { infection }\end{array}$ \\
\hline $\begin{array}{l}\text { Diagnostic } \\
\text { recommendation }\end{array}$ & $\begin{array}{l}\text { Recommends quantification of } \\
\text { leukocytes only in patients with } \\
\text { unexplained male infertility or for } \\
\text { evaluation of ART therapy options }\end{array}$ & $\begin{array}{l}\text { No recommendation for evaluation } \\
\text { of genital tract inflammation in } \\
\text { absence of symptoms }\end{array}$ & $\begin{array}{l}\text { Not included in standard semen } \\
\text { analysis }\end{array}$ \\
\hline $\begin{array}{l}\text { Treatment } \\
\text { recommendation }\end{array}$ & $N / A$ & $\begin{array}{l}\text { Therapy not indicated, but warrants } \\
\text { consideration in setting of } \\
\text { infertility }\end{array}$ & $\begin{array}{l}\text { Antibiotics may improve sperm } \\
\text { quality, but no evidence that } \\
\text { it increases the probability of } \\
\text { conception }\end{array}$ \\
\hline Level of evidence & $\mathrm{N} / \mathrm{A}$ & Treatment: $3 c$ & $\begin{array}{l}\text { Significance: } 3 \\
\text { Treatment: } 2 a\end{array}$ \\
\hline Most recent review & 2015 & 2011 & 2016 \\
\hline
\end{tabular}

This table reviews the four major urologic and andrologic associations and their diagnostic method, clinical significance, diagnostic and treatment recommendations regarding leukocytospermia. Also reported is the level of evidence and the most recent update to their guidelines. The international bodies that give recommendations and that are included in this table include the American Urologic Association (AUA), the American Society for Reproductive Medicine (ASRM), the Canadian Urological Association (CAU) and the European Association of Urology (EAU). N/A indicates this component was not discussed in the respective guideline article.

diagnostic recommendation. There is no mention of recommendations for treatment of this finding.

Much of this guideline is in reference to a single publication by Wolff and Anderson [12] in 1988. This randomized and controlled study aimed to evaluate immunohistochemistry's role in distinguishing leukocytes from the generic term "round cells." They found that by using staining with immunoperoxidase and immunohistochemistry, they could more accurately identify leukocytes within semen samples. Failure to use this method results in a possible overestimation of leukocyte numbers in semen.

Wolff and Anderson [12] also offered some findings in the link between LCS and unexplained male infertility. Evaluating semen samples of 51 infertile men and 17 proven fertile men, they report higher means and medians of leukocytes in the infertility group. Wolff and Anderson [12] also reported that a more reliable detection of leukocytes in semen may provide added value in the treatment of male infertility, especially with respect to the success of in vitro fertilization (IVF).

\section{American Society for Reproductive Medicine}

The ASRM uses an update of a largely similar ar- ticle as that of the AUA to establish guidelines on LCS. This article discusses the diagnosis, significance, and treatment of LCS in an identical paragraph as that in the AUA guidelines (above). Thus, no conflict of opinion was found between the two guidelines and no new information is offered.

\section{Canadian Urological Association}

The CUA only evaluates LCS within the context of their guidelines on prostatitis. Their guidelines make mention of genital tract inflammation and its significance specifically regarding chronic and asymptomatic prostatitis [13]. In this report, they make no mention of the recommended diagnostic procedure for semen analysis and quantification of leukocytes. They do not recommend further evaluation of the diagnosis of asymptomatic prostatitis, believing it to have no clinical relevance [13]. They do, however, admit that antimicrobial therapy can be considered in the context of infertility. This is given a level $3 \mathrm{C}$ recommendation. Unfortunately, there is no reference to evidence for this small point, and no further discussion on what treatment regimen would be preferred, leaving it up to the treating urologist's clinical judgement. 


\section{European Association of Urology}

The EAU offers by far the most comprehensive guidelines on this issue. Jungwirth et al [14] outline these recommendations. Similarly to the AUA and ASRM, they suggest wet mount microscopy followed with confirmatory peroxidase positive staining for accurate identification of leukocytes.

With respect to the clinical significance of LCS, EAU considers LCS to be taken as a sign of inflammation, and not a direct confirmation of bacterial or viral infection of the male genital tract. The guidelines state that urethritis and prostatitis have no clear association with male infertility using a level of evidence of 3 . Further, cited within is a publication by Tomlinson et al [15] that concludes that elevated leukocytes are not directly a cause of male infertility in isolation. In this study, 229 couples selected from an infertility clinic underwent semen analysis with leukocyte quantification confirmed by immunohistochemistry. Conception outcomes after 22 months without therapy were subsequently measured and related to semen parameters. Ultimately, there was no association between seminal leukocyte concentration and conception rate.

Nonetheless, treatment recommendations in this article suggest antibiotic therapy only. They note that only antibiotic therapy has been shown to eradicate the offending microorganisms (if present) and does not reduce the associated inflammatory alterations in urogenital secretions. They cite level of evidence of $2 \mathrm{a}$ for these assertions. However, they highlight that there is no evidence that antibiotic therapy will increase the probability of conception. Level $2 \mathrm{a}$ evidence is also attributed to this statement. This references two articles by Weidner et al [16] and Comhaire et al [17]. The study by Comhaire et al [17] evaluated 33 infertile couples in a double-blind comparison of treatment with doxycycline or a placebo control. They report no significant difference in conception rates per month between the two groups, indicating that infection may regress spontaneously and is not influenced by the antibiotic treatment.

\section{Review of clinical trials}

Since the guidelines are sparse and non-confirmatory, we performed a literature review as mentioned in the materials and methods above. Our search yielded eleven studies that evaluated outcomes of various therapies for LCS. Thirteen records were identified during our search. Four additional records were included as they were found in the sources of the guidelines studied and are very relevant to the evaluation of LCS treatment. Four records were removed through screening criteria due to non-English language or the study lacked treatment intervention, and one deemed ineligible as this study's seminal collection method varied so dramatically from that of the others studied. Specifically, it utilized vibratory stimulation and antegrade electroejaculation for collection of samples. Ultimately, 11 sources were evaluated and included within our study (Fig. 1). A comparison of each study's methods and results are summarized in Table 2. Of the eleven, seven compared antibiotic therapy [17-23], one evaluated anti-histamine therapy [24], one evaluated steroid treatment [25], and two evaluated non-steroidal anti-inflammatory drug (NSAID) treatment $[7,26]$.

In the group that compared antibiotic therapies, three showed significant improvement in the resolution rate of LCS over controls, three showed no significant differences, and one did not directly measure resolution of LCS. These studies differ on their assessment method and antibiotic therapy of choice. The intervention that was associated with significant resolution across studies was antibiotic treatment combined with frequent ejaculation seen in two studies [18,19]. Another correlation with successful therapy was duration of treatment. Three of the four studies that included treatment for at least one full month saw significant improvements in resolution rate. In addition, four studies measured qualitative and quantitative sperm parameters. Two of these showed significant improvement in these parameters following antibiotic therapy $[22,23]$. Finally, three studies evaluated pregnancy rate between antibiotic therapy and control groups, with one finding significant improvement within the treatment group. All three, however, found a higher rate of pregnancies in the treatment groups but did not have enough power with the sample sizes to find statistical significance.

Oliva and Multigner [24] studied the effect of ketotifen therapy, an anti-histamine, in an open, uncontrolled study. They found improved sperm motility, percentage of sperm with normal morphology, and a significant reduction in white blood cells (WBC) after the first four weeks of ketotifen treatment. They did not, however, note the utilization of immunohistochemistry or leukocyte-specific staining in order to confirm 


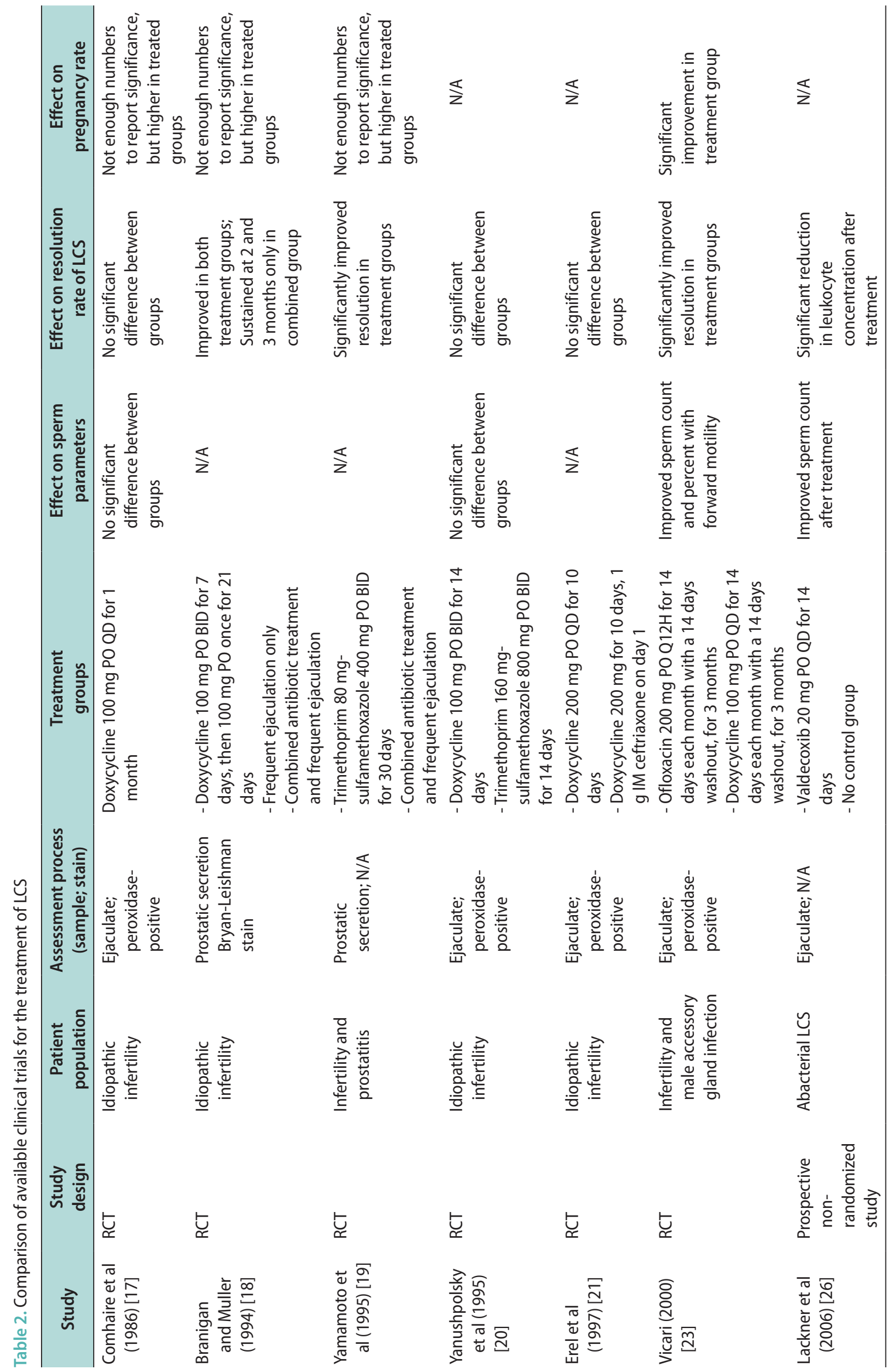




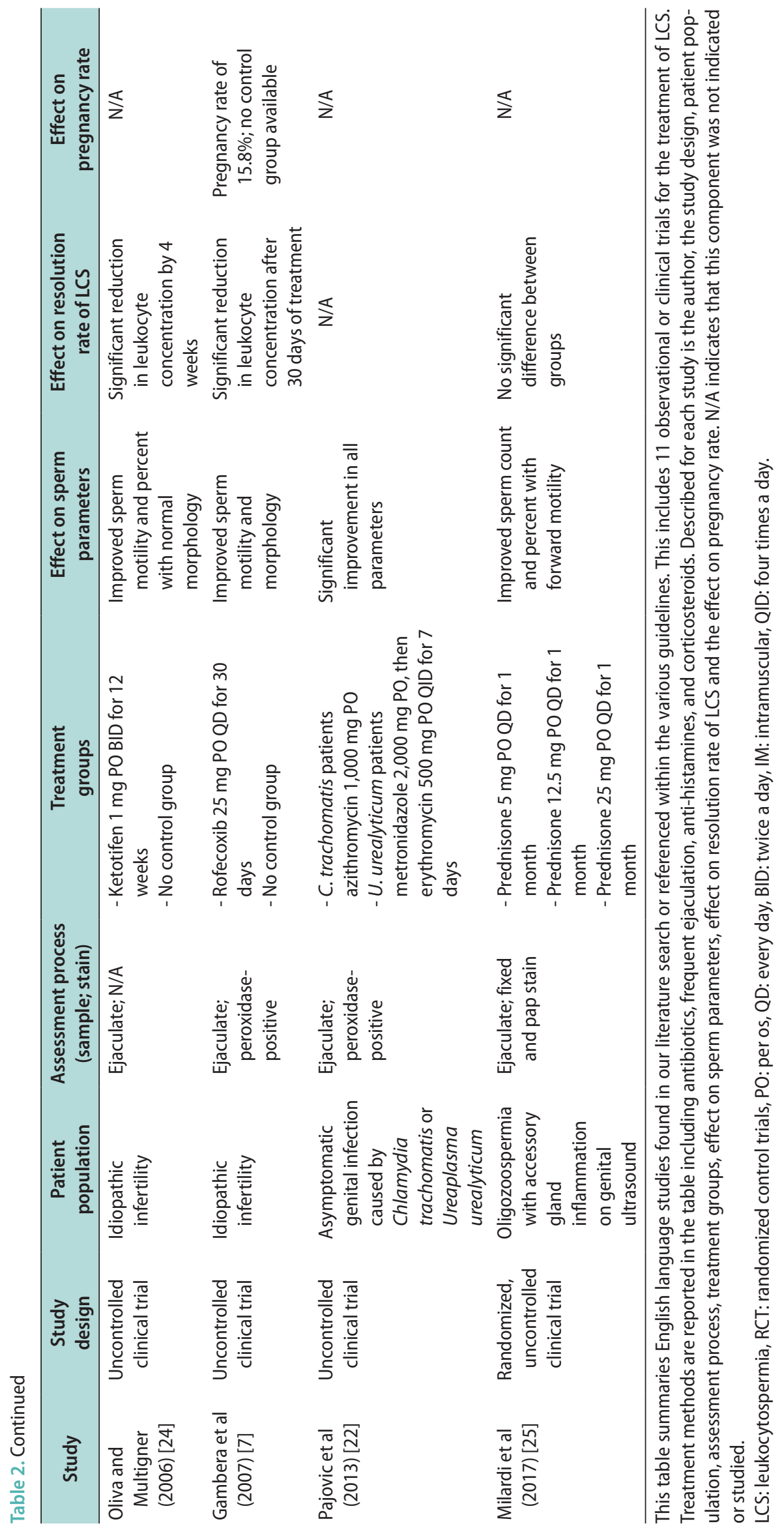


leukocyte count during semen analysis. While they did evaluate an objective measure, there was also no control group.

Milardi et al [25] studied the effect of steroid treatment on patients with LCS. They found no significant reduction in leukocyte count after one month of therapy with varying dosage groups of prednisone. They did find significant improvement in sperm count and percentage of sperm with forward motility, but these results should be considered separately as the population group consisted of men with oligozoospermia prior to intervention. A major limitation to this study is its lack of a control group, resulting in a higher risk of bias.

Finally, two studies evaluated the effect of NSAIDs as a therapy for LCS. Both were non-controlled and measured the effect of Cox-2 inhibitors on sperm quantity and quality before and after treatment. Both report results of significant improvement in LCS. Rofecoxib treatment resulted in improved sperm motility and morphology, whereas valdecoxib therapy only resulted in an improved sperm count. The lack of control groups, again, limits the strength of these findings.

\section{DISCUSSION}

LCS remains a confusing and controversial topic without a consensus on clinical significance nor treatment as evidenced by a comparison of guidelines worldwide. The above recommendations have been reviewed and updated within the last 10 years but still rely largely on evidence from the late 1980s and early 1990s. A review of the current observational studies and clinical trials validates this controversy. The only interventions that have consistently shown significant improvement in outcome appears to be a combination of antibiotic therapy and frequent ejaculation and for a duration of at least one month.

To demonstrate importance of some of the more recent findings in the management of LCS, we address three issues:

1) Lowering the WHO standard and redefining LCS.

2) Standardizing the treatment of LCS by giving longer duration of antibiotics.

3) Re-evaluating the success of antibiotic therapy with fertility rate as the endpoint.

\section{Definition of leukocytospermia}

Although the WHO standard for LCS has long been one million WBCs per $\mathrm{mL}$ of semen, recent evidence suggests that the presence of WBCs and the effect of subsequent generation of ROS can significantly affect sperm function at levels as low as $0.1 \times 10^{6} \mathrm{WBCs} / \mathrm{mL}$ $[27,28]$. Other studies have shown this critical point to exist at $0.2 \times 10^{6}$ [29-31] or even $0.5 \times 10^{6} \mathrm{WBCs} / \mathrm{mL}$ [32]. This suggests that by setting the standard at one million, many study participants may have been mischaracterized as unaffected by LCS when in fact they may have diminished fertility due to the presence of many WBC's. This would both understate the prevalence and effect of LCS as compared to unaffected individuals. Furthermore more patients may stand to benefit from evaluation and possible treatment of LCS based on these cut-off values.

Hamada et al [33] conducted a retrospective study using a proposed new threshold of $0.2 \times 10^{6} \mathrm{WBCs} / \mathrm{mL}$ for LCS to evaluate the effectiveness of antibiotic therapy. This study compared a treatment group of 34 men treated for 3 weeks with $100 \mathrm{mg}$ of doxycycline twice daily and a historical control group of 27 when treatment for LCS was not standard at this WBC level within their research facility. Like many previous studies, however, they were unable to show a significant improvement in qualitative sperm parameters. Unlike other studies, however, they did find an increase in the natural pregnancy rate among those in the treatment group. They report rates of $47 \%$ versus $20 \%$ in the treatment and control groups, respectively. Additionally, the authors argue extending the treatment to 3 months or longer may benefit qualitative sperm parameters.

\section{Duration of antibiotic treatment and pregnancy rate as an end-point of the treatment for leukocytospermia}

Our review of the literature shows that few randomized controlled trials (RCT) measure pregnancy rate when comparing antibiotic therapy to control [7,9,1719,23]. Among these, only one showed a significant improvement in pregnancy rate with antibiotic treatment [23]. This study included treatment with doxycycline or ofloxacin and also continued treatment for a longer period of 3 months. This is corroborated by the finding that the three studies that found significant reduction in LCS all utilized treatment for at least one month. Additionally, three RCT's demonstrated an increased number of pregnancies in the treatment groups, how- 
ever there not a statistically significant difference in pregnancy rate [17-19]. While there is still no definitive evidence that antibiotics result in higher pregnancy rates among patients with LCS, there are more recent studies that demonstrate a potential need to increase the duration of antibiotic treatment, combine antibiotics with frequent ejaculation, and potentially lower the threshold for LCS itself. Furthermore, there is a clear dearth of high quality evidence for the role of alternative anti-inflammatory therapy. Of the three studies evaluated, none were RCT and no such studies were found during our literature search. Due to the role of inflammation in decreasing semen quality, this area of therapy warrants further investigation.

Finally, one area that is linked to LCS but receives little consideration in the current proposed guidelines is the lack of antibiotic treatment effect on ART outcomes. For men with infertility, there is no significant difference in success rate for both IVF and intracytoplasmic sperm injection (ICSI) between leukocytospermic and non-leukocytospermic groups [34]. Additionally, by shifting the threshold from $2 \times 10^{6}$ to $0.2 \times 10^{6} \mathrm{WBCs} /$ $\mathrm{mL}$, the success of IVF and ICSI, did not significantly change. Ultimately, this could indicate that no antibiotic therapy trial is necessary in the setting of ART. This could possibly be due to semen processing done prior to the sample being used. However, many fertility clinics still use short courses of antibiotics in male sperm donors with known LCS.

\section{CONCLUSIONS}

LCS continues to be a perplexing topic for urologists and andrologists. A large base of contradictory evidence exists for the clinical significance or irrelevance of LCS. Guidelines on the topic, thus, remain equally noncommittal. In comparing them, the major differences are the AUA's link of LCS to an infection whereas the EAU suggests it likely merely indicates inflammation. There has, however, been consistent evidence that the ROS species and presence of leukocytes in semen still impairs sperm quality by quantitative measures. Furthermore, recent studies have shown that the duration of treatment and the definition of LCS may impact the success of antibiotic therapy in improving rates of natural conception. These changes should thus be considered in the guidelines of various organizations around the world. Finally, the presence of LCS in the setting of ART seems to be a non-factor, however many fertility centers do treat the males with a short course of antibiotics if they are found to have LCS.

\section{ACKNOWLEDGEMENTS}

The authors would like to thank Karen Boling for her assistance in coordinating, supporting and organizing urological education and research as the medical student and urology residency program coordinator.

\section{Disclosure}

The authors have no potential conflicts of interest to disclose.

\section{Author Contribution}

Brunner RJ was the principal author who contributed in all facets of the manuscript: initial concept, design, acquisition of literature data, analysis, primary drafter of the article, and manuscript editions. Demeter JH made contributions to the analysis of articles reviewed, writing and editing of the manuscript. Sindhwani P contributed to the concept, design, as well as the analysis of the articles reviewed. He partook in critical revisions and editing of the manuscript.

\section{REFERENCES}

1. Gnoth C, Godehardt E, Frank-Herrmann P, Friol K, Tigges J, Freundl G. Definition and prevalence of subfertility and infertility. Hum Reprod 2005;20:1144-7.

2. Thonneau P, Marchand S, Tallec A, Ferial ML, Ducot B, Lansac J, et al. Incidence and main causes of infertility in a resident population $(1,850,000)$ of three French regions (1988-1989). Hum Reprod 1991;6:811-6.

3. Agarwal A, Mulgund A, Hamada A, Chyatte MR. A unique view on male infertility around the globe. Reprod Biol Endocrinol 2015;13:37.

4. Sandoval JS, Raburn D, Muasher S. Leukocytospermia: overview of diagnosis, implications, and management of a controversial finding. Middle East Fertil Soc J 2013;18:129-34.

5. Bachir BG, Jarvi K. Infectious, inflammatory, and immunologic conditions resulting in male infertility. Urol Clin North Am 2014;41:67-81.

6. Shy KK, Stenchever MA, Muller CH. Sperm penetration assay and subsequent pregnancy: a prospective study of 74 infertile men. Obstet Gynecol 1988;71:685-90.

7. Gambera L, Serafini F, Morgante G, Focarelli R, De Leo V, 
Piomboni P. Sperm quality and pregnancy rate after COX-2 inhibitor therapy of infertile males with abacterial leukocytospermia. Hum Reprod 2007;22:1047-51.

8. Domes T, Lo KC, Grober ED, Mullen JB, Mazzulli T, Jarvi $\mathrm{K}$. The incidence and effect of bacteriospermia and elevated seminal leukocytes on semen parameters. Fertil Steril 2012; 97:1050-5.

9. Jung JH, Kim MH, Kim J, Baik SK, Koh SB, Park HJ, et al. Treatment of leukocytospermia in male infertility: a systematic review. World J Mens Health 2016;34:165-72.

10. World Health Organization. WHO laboratory manual for the examination and processing of human semen. 5th ed. Geneva: World Health Organization; 2010.

11. Jarow J, Sigman M, Kolettis PN, Lipshultz LR, McClure RD, Nangia AK, et al. The optimal evaluation of the infertile male: AUA best practice statement. Linthicum: American Urological Association, Inc.; 2011.

12. Wolff $\mathrm{H}$, Anderson DJ. Immunohistologic characterization and quantitation of leukocyte subpopulations in human semen. Fertil Steril 1988;49:497-504.

13. Nickel JC. Prostatitis. Can Urol Assoc J 2011;5:306-15.

14. Jungwirth A, Giwercman A, Tournaye H, Diemer T, Kopa Z, Dohle G; European Association of Urology Working Group on Male Infertility, et al. European Association of Urology guidelines on male infertility: the 2012 update. Eur Urol 2012; 62:324-32.

15. Tomlinson MJ, Barratt CL, Cooke ID. Prospective study of leukocytes and leukocyte subpopulations in semen suggests they are not a cause of male infertility. Fertil Steril 1993;60:1069-75.

16. Weidner W, Krause W, Ludwig M. Relevance of male accessory gland infection for subsequent fertility with special focus on prostatitis. Hum Reprod Update 1999;5:421-32.

17. Comhaire FH, Rowe PJ, Farley TM. The effect of doxycycline in infertile couples with male accessory gland infection: a double blind prospective study. Int J Androl 1986;9:91-8.

18. Branigan EF, Muller CH. Efficacy of treatment and recurrence rate of leukocytospermia in infertile men with prostatitis. Fertil Steril 1994;62:580-4.

19. Yamamoto M, Hibi H, Katsuno S, Miyake K. Antibiotic and ejaculation treatments improve resolution rate of leukocytospermia in infertile men with prostatitis. Nagoya J Med Sci 1995;58:41-5.

20. Yanushpolsky EH, Politch JA, Hill JA, Anderson DJ. Antibiotic therapy and leukocytospermia: a prospective, randomized, controlled study. Fertil Steril 1995;63:142-7.

21. Erel CT, Sentürk LM, Demir F, Irez T, Ertüngealp E. Antibiotic therapy in men with leukocytospermia. Int J Fertil Womens Med 1997;42:206-10.
22. Pajovic B, Radojevic N, Vukovic M, Stjepcevic A. Semen analysis before and after antibiotic treatment of asymptomatic Chlamydia- and Ureaplasma-related pyospermia. Andrologia 2013;45:266-71.

23. Vicari E. Effectiveness and limits of antimicrobial treatment on seminal leukocyte concentration and related reactive oxygen species production in patients with male accessory gland infection. Hum Reprod 2000;15:2536-44.

24. Oliva A, Multigner L. Ketotifen improves sperm motility and sperm morphology in male patients with leukocytospermia and unexplained infertility. Fertil Steril 2006;85:240-3.

25. Milardi D, Luca G, Grande G, Ghezzi M, Caretta N, Brusco $\mathrm{G}$, et al. Prednisone treatment in infertile patients with oligozoospermia and accessory gland inflammatory alterations. Andrology 2017;5:268-73.

26. Lackner JE, Herwig R, Schmidbauer J, Schatzl G, Kratzik C, Marberger M. Correlation of leukocytospermia with clinical infection and the positive effect of antiinflammatory treatment on semen quality. Fertil Steril 2006;86:601-5.

27. Henkel R, Kierspel E, Stalf T, Mehnert C, Menkveld R, Tinneberg HR, et al. Effect of reactive oxygen species produced by spermatozoa and leukocytes on sperm functions in nonleukocytospermic patients. Fertil Steril 2005;83:635-42.

28. Sharma RK, Pasqualotto AE, Nelson DR, Thomas AJ Jr, Agarwal A. Relationship between seminal white blood cell counts and oxidative stress in men treated at an infertility clinic. J Androl 2001;22:575-83.

29. Menkveld R, Kruger TF. Sperm morphology and male urogenital infections. Andrologia 1998;30 Suppl 1:49-53.

30. Punab M, Lõivukene K, Kermes K, Mändar R. The limit of leucocytospermia from the microbiological viewpoint. Andrologia 2003;35:271-8.

31. Lackner J, Schatzl G, Horvath S, Kratzik C, Marberger M. Value of counting white blood cells (WBC) in semen samples to predict the presence of bacteria. Eur Urol 2006;49:148-52; discussion 152-3.

32. Thomas J, Fishel SB, Hall JA, Green S, Newton TA, Thornton SJ. Increased polymorphonuclear granulocytes in seminal plasma in relation to sperm morphology. Hum Reprod 1997; 12:2418-21.

33. Hamada A, Agarwal A, Sharma R, French DB, Ragheb A, Sabanegh ES Jr. Empirical treatment of low-level leukocytospermia with doxycycline in male infertility patients. Urology 2011;78:1320-5.

34. Ricci G, Granzotto M, Luppi S, Giolo E, Martinelli M, Zito G, et al. Effect of seminal leukocytes on in vitro fertilization and intracytoplasmic sperm injection outcomes. Fertil Steril 2015; 104:87-93. 